\title{
Low Cost Laboratory Type Signal Generator Using DDS Method
}

\author{
Süleyman Bilgin ${ }^{a}$, Yavuz $\ddot{U}_{s e r}{ }^{b^{*}}$ and Mustafa Oktay $^{c}$ \\ ${ }^{a, b, c}$ Akdeniz University, Faculty of Engineering, Electrical and Electronics Engineering, Antalya, Turkey \\ ${ }^{*}$ E-mail address: yuser@akdeniz.edu.tr
}

Received date: November 2016

Accepted date: December 2016

\begin{abstract}
Everyone who is an amateur or student interested in electricity or electronics needs a signal generator that can produce different types of signals that must be located at the front edge. This requirement can often be met by equipment in the laboratories or signal generators received at high prices under the project. This study focuses on the design of a low cost signal generator having high accuracy and sensitivity. In previous studies, DDS (Direct Digital Synthesis) method was implemented with FPGA and other high cost microcontrollers. With this study, the design of the high-accuracy signal generator has been made simpler and more cost-effective thanks to the PIC microcontroller, which can be found easily in the market. The device designed in the study is a digital signal generator that produces sine, triangle and square wave which are created using decimal numbers in the microcontroller and converted to analog signal via DAC (Digital to Analog Converter). Also, the digital potentiometer on the device, which is used as feedback in the circuit, allows us to control the output signal. This study provides a solution for producing a low cost laboratory type signal generator having high sensitivity, however this solution is not a new method. Satisfactory results have been achieved from the implementation circuit.
\end{abstract}

Keywords: Signal Generator, Direct Digital Synthesis, Phase Circle.

\section{Introduction}

There are some problems such as non-precision calibration adjustment, false wave generation, frequency shift in analogue signal generators. The solution of such problems is to use a digital signal source in the designs. In this way, the desired wave types can be seen more accurately and steadily. PIC microcontrollers are used for the digital signal source in this study. The frequency and amplitude values of the signal can be changed easily within certain limits on the designed device. The designed signal generator generates the desired waveform up to $5 \mathrm{KHz}$. This value can be raised to higher frequencies with a simple frequency multiplier. There are two known methods for generating signals with a microcontroller. These are PWM (Pulse Width Modulation) and DDS methods.

In the PWM method, the PWM signal is generated by varying the duty cycle value. Each generated PWM signal is sent to the integral receiver circuit and converted to a voltage level here. The output signal is obtained after low pass filtering. The values of the low-pass filter are adjusted depending on the maximum frequency of the output signal. The integral operation of the PWM method causes frequency shifts. This is not a desirable process. In the DDS method, the digital signal to be generated is stored into the register of the microcontroller. These stored values are sent from selected port to the output of DAC (Digital Analog Converter) module respectively after processing of them 
using DDS method. Then, the generated analog signal is passed through a low pass filter circuit as in the PWM method to obtain the desired signal. At this point, the shape of the generated signal is clearer and its frequency is stabilized. Shoucheng Ding et. all have designed a dall-digital frequency synthesizer technology with FPGA. The frequency was adjustable through10-bit phase accumulator and the analog multiplier achieved amplitude modulation. And they used the direct numerical frequency synthesizer based on FPGA[1]. Pranjal A. Charde et. all made Sine, Cosine, Square \& Saw tooth waveforms generator by using DDS Multiple waveform generator simulation in Multisim Simulation Program. This design is useful for measuring signal values at high frequency upto $100 \mathrm{MHz}$ and the power consumption is reduce up to $0.02720 \mathrm{~W}$. [2] Cao Xiaodong et. all designed compact direct digital frequency synthesizer (DDFS) for system-on-chip (SoC). ROM size is reduced 98\% using QLA technique and QE-ROM technique. Their DDFS chip with core area of $1.6 \mathrm{~mm} 2$ has been successfully fabricated using standard $0.35 \mathrm{um}$ CMOS process. And it consumes $167 \mathrm{~mW}$ at 3.3V and its SFDR is $61 \mathrm{~dB}$ [3]. Liuying, Limin realized a function signal generator, with MSP430 as control core, based on DDS chip AD9850, with adjustable frequency of the sine wave signal, square wave and triangular wave signal. There is no obvious distortion on produced output signals [4].Liu Ke et. all designed the method is based on characteristic of LC parallel circuits that it improved the amplitude-frequency characteristic of output signal. By this method, amplitude attenuation caused by DAC is compensated; amplitude frequency characteristic of DDS signal is improved [5].

This study consists of 3 parts. While the first part of the study is interested in the introduction of the method, the second part is gives an information about DDS method the obtaining of the output signal using DAC, low pass filter and digital potentiometer. In the last section, it is given application phase, and the output signal graphics.

\section{2.a DDS METHOD (Direct Digital Synthesis)}

The PWM fixed period square wave signal is generated by changing the duty cycle ratio. The higher the duty cycle rate supplies the signal having higher output voltage. This voltage value is shown in figure1 [6].

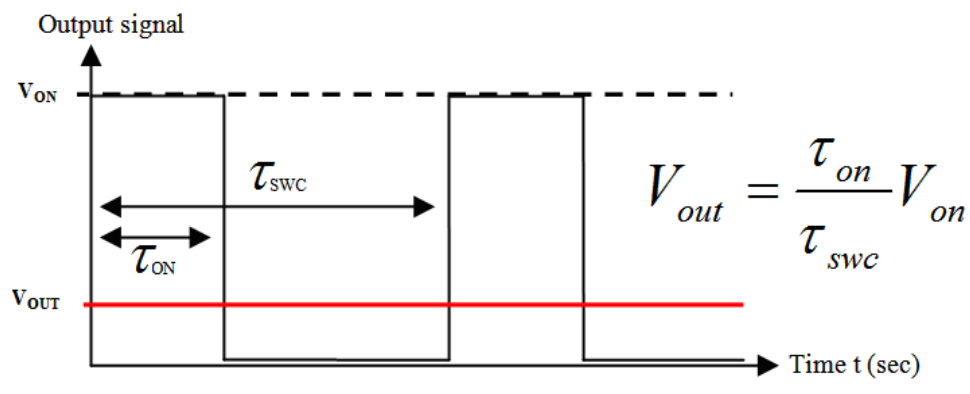

Fig. 1. Signal generation with PWM technique

Von is the upper value of the PWM signal. The parameter $\tau_{\text {on }}$ is the time that the squared wave remains at the maximum level and $\tau_{\text {swc }}$ is the period of the square wave. It is necessary to increase the frequency of the PWM signal be able to provide higher frequencies. This means an extra cost.

In the DDS method, more accurate and faster results are obtained. Figure 2 shows the DDS block diagram [7]. The digital values of the signal to be generated are stored in the register of the microcontroller. These values are assigned related to the number of bits used in the microcontroller. 
Table 1 shows a sine signal table with 8 bits $(28=256)$ and a step interval as average of 10 . This table is drawn based on the phase circle and the formula is $127+127 * \sin (((2 *$ pi $) / 360) *$ Degree $)$. The number " 127 " in the form is equal to the half of the maximum decimal number that can be taken from an 8 -bit port $(256 / 2=128)$. Each angle value of the sine is multiplied by the maximum decimal value in the microcontroller. In order to make the output results as positive, the DC offset value is

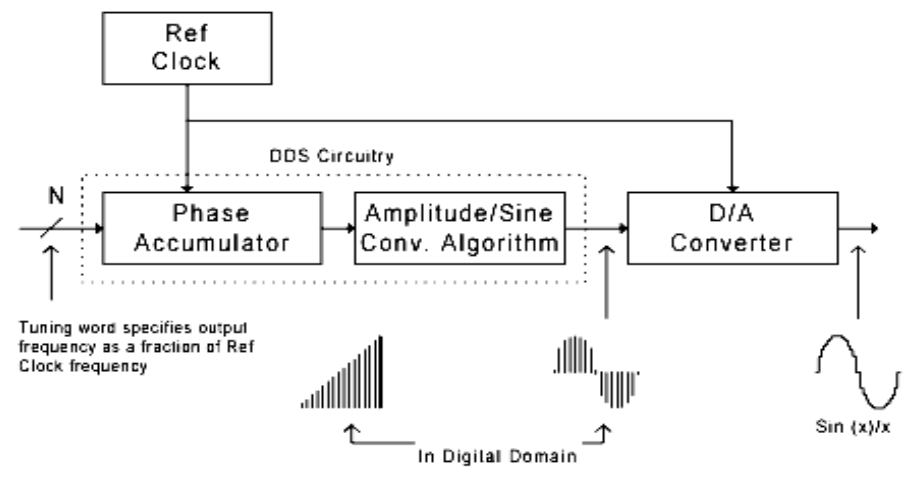

Fig. 2. Direct Digital Synthesis Method

added to the signal, where the signal changes into a sinusoidal signal ranging from 0 to 255 .

Table 1. Digital values of signal that is desired to produce

\begin{tabular}{c|ccc} 
Degree & $\begin{array}{c}\text { Formula } \\
\text { Result }\end{array}$ & Degree & $\begin{array}{c}\text { Formula } \\
\text { Result }\end{array}$ \\
\hline $\mathbf{1 0}$ & 127,000 & $\mathbf{1 9 0}$ & 127,000 \\
$\mathbf{2 0}$ & 149,053 & $\mathbf{2 0 0}$ & 104,947 \\
$\mathbf{3 0}$ & 170,437 & $\mathbf{2 1 0}$ & 83,563 \\
$\mathbf{4 0}$ & 190,500 & $\mathbf{2 2 0}$ & 63,500 \\
$\mathbf{5 0}$ & 208,634 & $\mathbf{2 3 0}$ & 45,366 \\
$\mathbf{6 0}$ & 224,288 & $\mathbf{2 4 0}$ & 29,712 \\
$\mathbf{7 0}$ & 236,985 & $\mathbf{2 5 0}$ & 17,015 \\
$\mathbf{8 0}$ & 246,341 & $\mathbf{2 6 0}$ & 7,659 \\
$\mathbf{9 0}$ & 252,071 & $\mathbf{2 7 0}$ & 1,929 \\
$\mathbf{1 0 0}$ & 254,000 & $\mathbf{2 8 0}$ & 0,000 \\
$\mathbf{1 1 0}$ & 252,071 & $\mathbf{2 9 0}$ & 1,929 \\
$\mathbf{1 2 0}$ & 246,341 & $\mathbf{3 0 0}$ & 7,659 \\
$\mathbf{1 3 0}$ & 236,985 & $\mathbf{3 1 0}$ & 17,015 \\
$\mathbf{1 4 0}$ & 224,288 & $\mathbf{3 2 0}$ & 29,712 \\
$\mathbf{1 5 0}$ & 208,634 & $\mathbf{3 3 0}$ & 45,366 \\
$\mathbf{1 6 0}$ & 190,500 & $\mathbf{3 4 0}$ & 63,500 \\
$\mathbf{1 7 0}$ & 170,437 & $\mathbf{3 5 0}$ & 83,563 \\
$\mathbf{1 8 0}$ & 149,053 & $\mathbf{3 6 0}$ & 104,947
\end{tabular}

Values in the microprocessor is also known as phase circle in the Figure 3 [8] . 


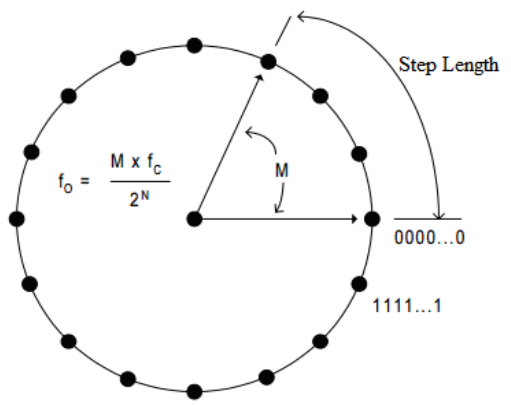

Fig. 3. Phase Circle

In the DDS method, This phase circle could be produced sinus signal. Then, step values in the circle is recorded up to decimal points.

Each recorded value is routed to ports of microprocessor respectively as a result is signal developed. Speed that each value has is responsible for change in the signal frequency. In the study, we determine the signal frequency using Timer 1 interruption. If the required signal frequency increases, the number of samples in the microprocessors decreases. In this study, the sampling number is 36 for $5 \mathrm{KHz}$. The DDS method program codes for the sinus signal we mentioned above as fallows.

Table 2. DDS Method Program Codes For The Sinus Signal

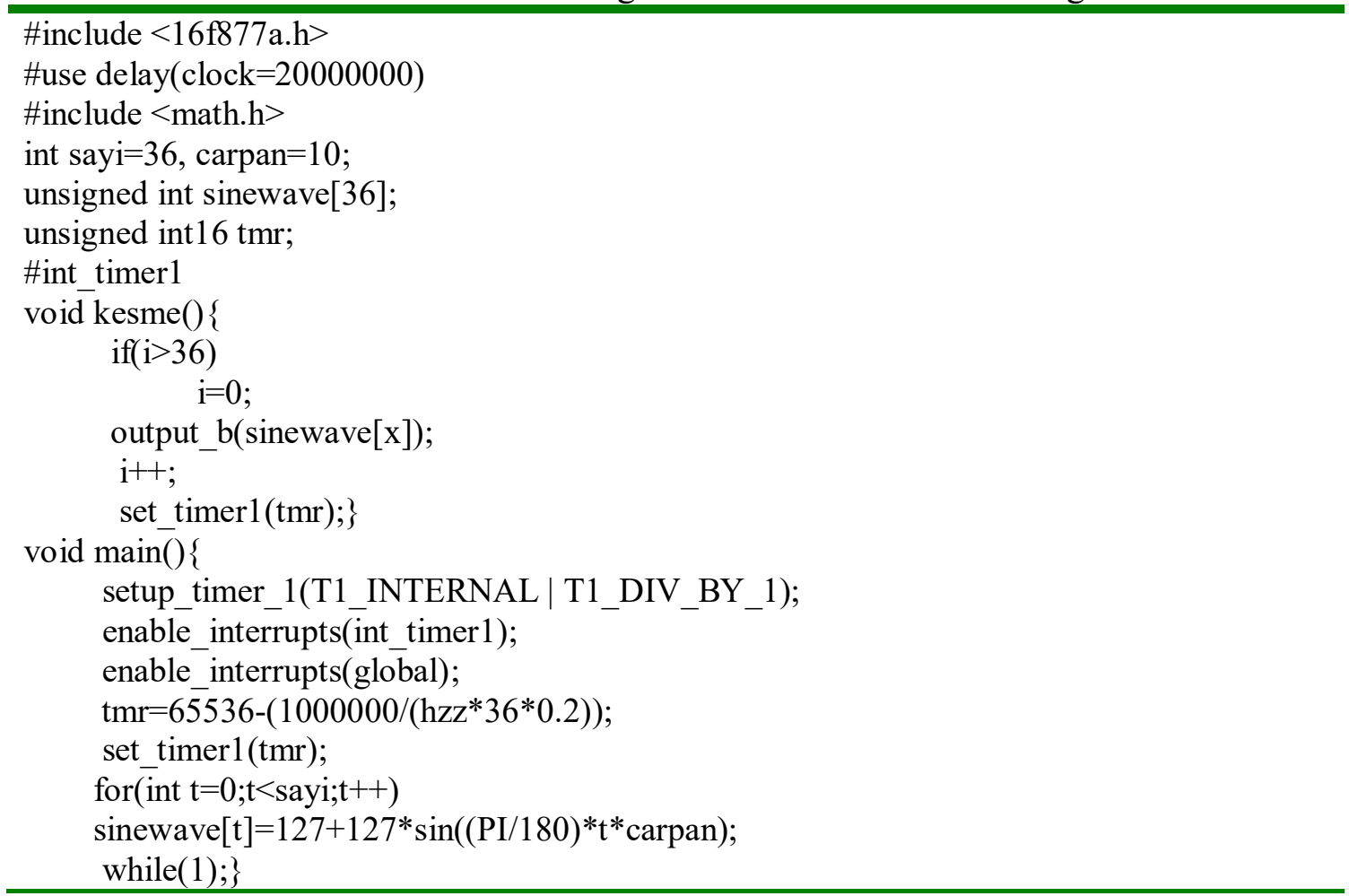

\section{2.b Digital Analog Converter}

Analog signal is determined using the conventional R-2R method as shown in the Figure 4.[9] 


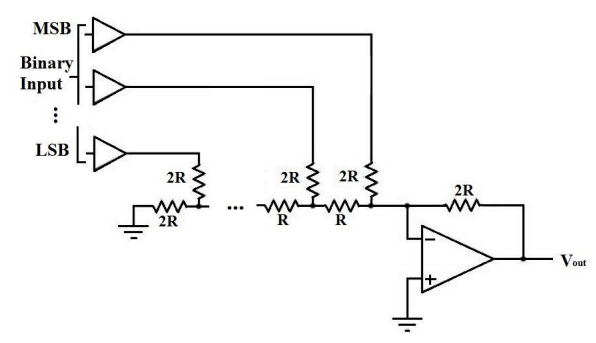

Fig. 4. R-2R Method

Maximum output voltage is $5 \mathrm{v}$. Output voltage is formulated below. We denote $\mathrm{V}_{\text {ref }}$ as the maximum voltage from microprocessor port, VAL as digital data that the microprocessor applies to the DAC module, and $\mathrm{N}$ as the number there is apply to the DAC module.[10]

$$
\mathrm{V}_{\text {out }}=\mathrm{V}_{\text {ref }} \times \mathrm{VAL} / 2^{\mathrm{N}}
$$

\section{2.c Low Pass Filter Module and Digital Potentiometer}

The signal in the microprocessor changes rapidly at the output DAC module, therefore we should filter the signal A simple low pass filter as shown in figure 5 cloud be used for this purpose (Figure 5) $[11]$.

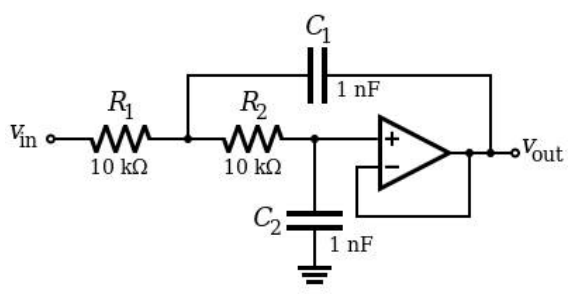

Fig. 5. Low Pass Filter values

DS1868-10 digital potentiometer integrated is utilized to validate the output signal. Digital code of the signal in the -microprocessor is both send to the DAC and DS1868-10. Output resistant and the voltage change proportionally to Digital data that has been sent to DS1868-10These data are checked against the output signal by applying them to the analog entry of the controller. The resistance interval of DS1868-10 integrated is measured by dividing resistance value to 256 . $10 \mathrm{~K} \Omega / 256=39,0625 \Omega$ is obtained.

\section{The Application Phase}

In this study, Microchip's PIC16F877A integrated circuit is used as a microcontroller. An output signal having a maximum $5 \mathrm{KHz}$ frequency values can be obtained from the circuit designed in the study. However, a PIC microcontroller with higher oscillator frequency can be needed to obtain higher frequencies. The microcontroller oscillator frequency in this study is $20 \mathrm{MHz}$. As another method, a desired frequency can be obtained with a frequency multiplier / divider circuit. [12] Figure 6 presents the circuit schematic of the study. Figures 7-8-9-10 present applied circuit and resulting signals. 


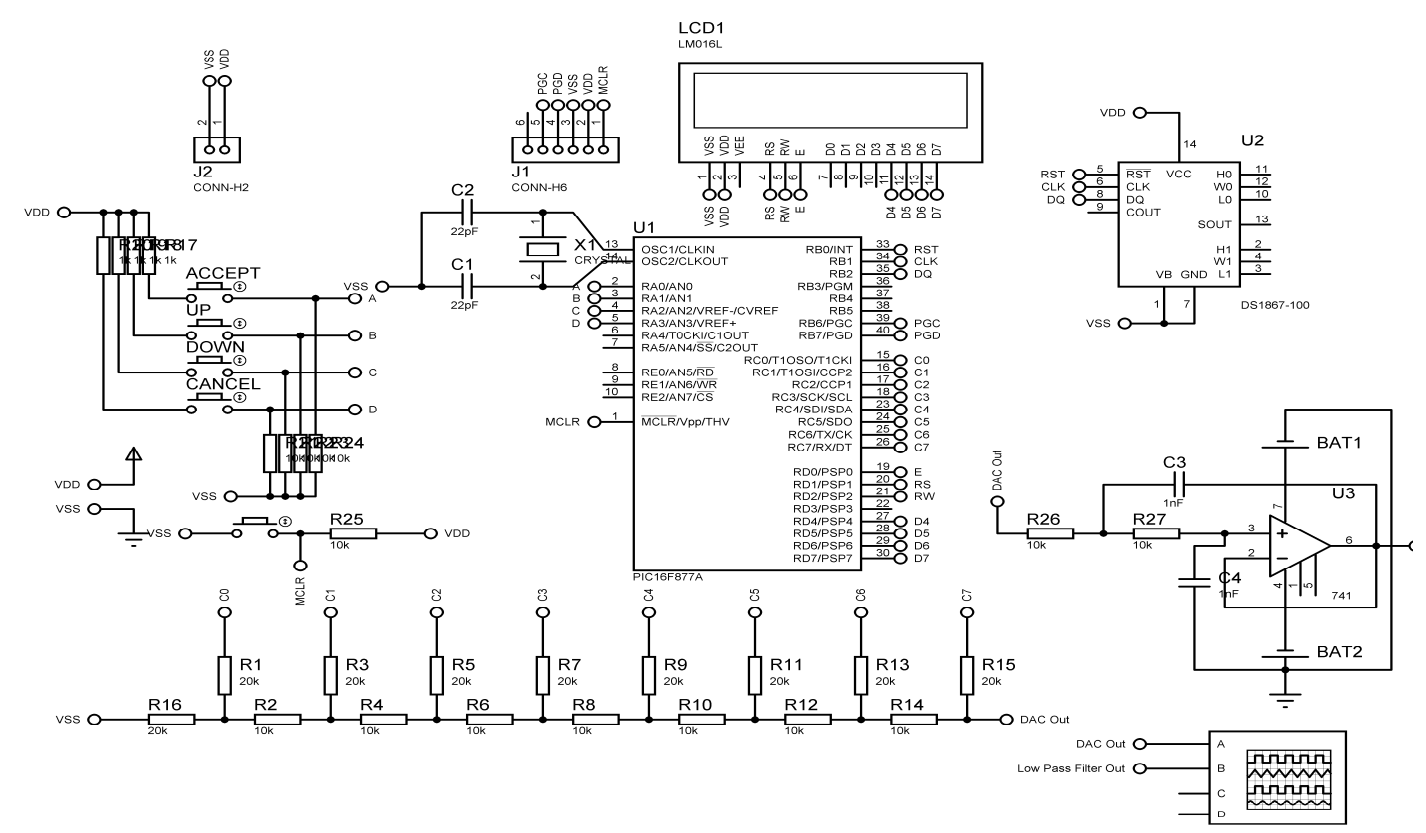

Fig. 6. Circuit Schema

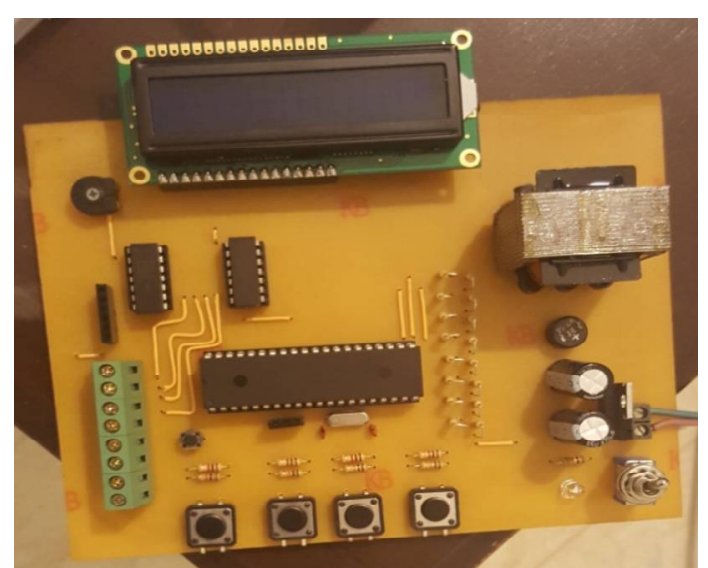

Fig. 7. Applied Circuit

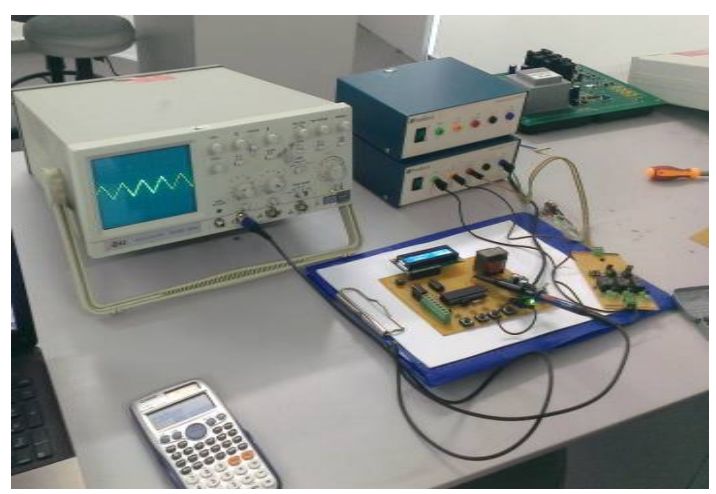

Fig. 9. Triangle wave output

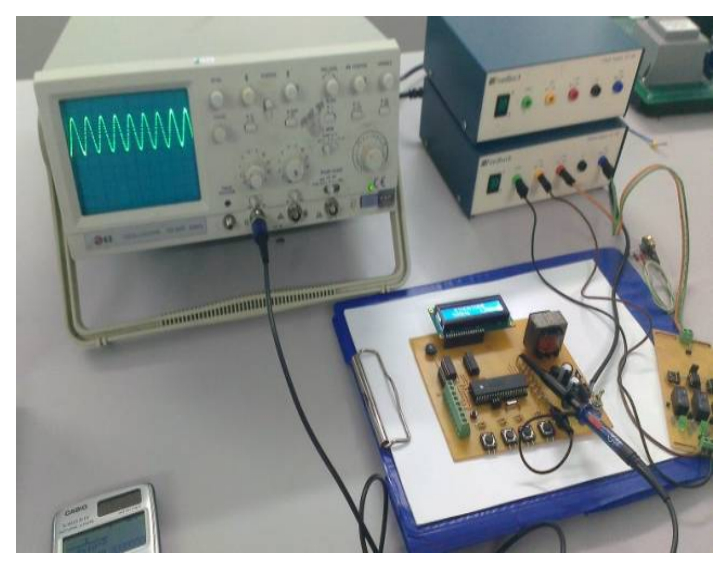

Fig. 8. Sinus wave output

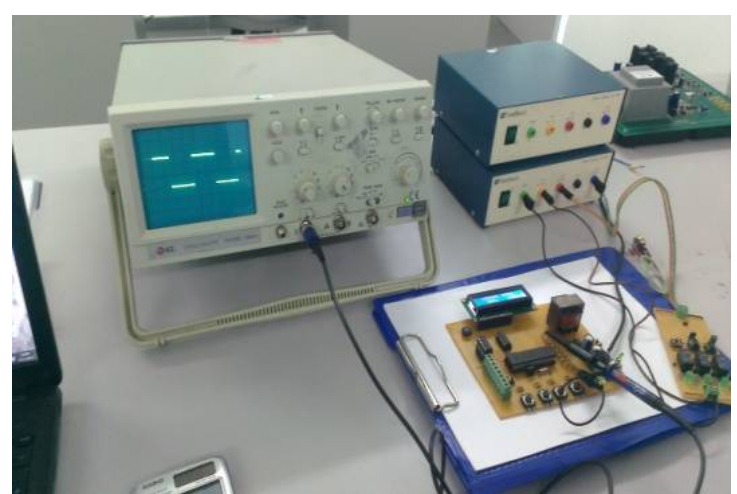

Fig. 10. Square wave output

\section{Conclusion}

In this study, we design a low cost high accuracy signal generator using the DDS method and PIC microcontroller. The output signals could be sinus, triangle and square wave up to $5 \mathrm{KHz}$. The 
maximum output value is $5 \mathrm{v}$. Frequency could be increased using multiplier. The results suggest that the error is less than $1 \%$ and there are no slips in the output frequency. This error is $5 \%$ in the PWM method. In edition, our method could produce the output value accurately without the need of high frequencies.

\section{Acknowledgments}

The study has been supported by the Research Project Department of Akdeniz University, Antalya, Turkey. This study is a part of studies held by Akdeniz University Industrial and Medical Applications Microwave Research Center (IMAMWRC).

\section{References}

[1] Shoucheng Ding, Aimin An and Xinke Gou, "Digital Waveform Generator Basedon FPGA", Research Journal of Applied Sciences, Engineering and Technology, July 15, 2012.

[2] Pranjal A. Charde1, Prof. P.R. Lakhe, Akshay P. Nanote, Design of Multiple Waveform Generator \& Frequency Counter Based On DDS, International Research Journal of Engineering and Technology,3(7), 2016.

[3] Cao Xiaodong, Ni Weining, Yuan Ling, Hao Zhikun, Shi Yin, A Compact Direct Digital Frequency Synthesizer for System-on-chip, Solid-State and Integrated-Circuit Technology,IEEE, 2008.

[4] Liuying. Limin, Function Signal Generator, Fourth International Conference on Digital Manufacturing \& Automation, IEEE, 2013.

[5] Liu Ke, Tian Shulin, Xiao Yindong, A Method Of Amplitude-Frequency Characteristic Compensation About DDS Signal Source, The Eighth International Conference on Electronic Measurement and Instruments, 2007.

[6] Ziad Nouman, Bohumil Klima, Jan Knobloch, Generating PWM Signals With Variable Duty From 0 percent to 100 percent Based Fpga Spartan3an, Elektrorevue ISSN1213 - 1539, 4(4), ,2013.

[7] A Technical Tutorial on Digital Signal Synthesis, Analog Devices, Inc.1999.

[8] M. Y. Chua, V. C. Koo," FPGA-BASED CHIRP GENERATOR FOR HIGH RESOLUTION UAV SAR" Progress In Electromagnetics Research, PIER 99, 71-88, • January 2009

[9] Anuradha S. Kherde, P.R.Gumble, A Review of Various Trends of Digital-To-Analog Converter with Performance Characteristics and Behavioral Parameters, International Journal of Advanced Research in Computer Science and Software Engineering, 3( 4), 2013.

[10] Anuradha S. Kherde, Pritesh R. Gumble, An Efficient Design of R-2R Digital to Analog Converter with Better Performance Parameter in (90nm) $0.09-\mu \mathrm{m}$ CMOS Process, International Journal of Innovative Technology and Exploring Engineering (IJITEE) ISSN: 2278-3075, 3(7), 2013. [11] Jim Karki, "Active Low-Pass Filter Design", AAP Precision Analog, Application Report, September 2002.

[12]PIC16F87XA Datasheet, Microchip Technology Inc. ,2003.

[13] Dual Digital Potentiometer Chip,Dallas Semiconductor, www.dalsemi.com datasheet number: 100899.

[14] TT electronics plc LIT-AN-R2RLADDER, "R/2R LADDER NETWORKS - Application Note" Issue 2.

[15] Vinaya Skanda, "Sine Wave Generator Using Numerically Controlled Oscillator Module", Microchip Technology Inc. 